\title{
SPINAL CORD PROTECTION DURING THORACOABDOMINAL ANEURYSM RESECTION
}

\author{
Paul A. Wojewski, Rapid City, S.D.
}

Paraplegia is a devastating complication after thoracoabdominal aneurysm resection. Multiple methods of spinal protection have been explored. ${ }^{1-9}$ At present, no reliable method of protecting the spine from ischemic injury exists. I am describing here two cases of thoraco abdominal aneurysm resection with selective cooling of the spinal cord. While the aorta was crossclamped, continuous lavage of the spinal cord with cold normal saline solution was introduced into the subarachnoid space by lumbar puncture. No neurologic sequela developed after crossclamping of the aorta for 58 minutes in case 1 and 36 minutes in case 2 .

Case 1. A 72-year-old woman was referred to me with a symptomatic thoracoabdominal aneurysm. Angiography showed a large arteriosclerotic aneurysm originating in the distal aortic arch, propagating throughout the thoracic aorta, and involving most of the abdominal aorta below the renal arteries. Spinal cord protection protocol was approved by the Institutional Review Committee of the Rapid City Regional Hospital.

On the day of the operation, the patient was taken first to the angio suite for placement of catheters to the subarachnoid space. The procedure was performed with the patient under heavy sedation by an invasive radiologist. The patient was placed prone on a fluoroscopic table with tilt capabilities. The subarachnoid space was entered at the L2-3 level with midline stick with a 20-gauge spinal needle (Fig. 1). The Cook micropuncture system guidewire (Cook Incorporated, Bloomington, Ind.) was then placed through the spinal needle into the subarachnoid space and advanced upward in the midline. After the dilating catheter was positioned ventral within the subarachnoid space, the 0.035 Tad II wire with 0.018 tip (Advanced Cardiovascular Systems, Inc., Santa Clara, Calif.) was passed. The Tad wire is steerable and the 0.018 tip helped avoid damage to nerve roots or cord while the wire was being advanced. The Tad wire was positioned above T5 ventral to the spinal cord. A 5F straight flush catheter was placed over the wire and positioned above T5. By means of the procedure just described, the second $5 \mathrm{~F}$ catheter was placed at the level of the conus medullaris. The catheter should have four to five additional side holes placed at its tip. The entrance into the subarachnoid space for this second catheter should be, if possible, one level below the entrance of the first catheter. The third catheter was placed at the next inferior level with the tip

From the Cardiac Vascular and Thoracic Surgery P.C., 350 Elk St., Suite B, Rapid City, S.D.

J Thorac CARdiovasc Surg 1995;109:1244-6

Copyright (C) 1995 by Mosby-Year Book, Inc.

$0022-5223 / 95 \$ 3.00+0 \quad \mathbf{1 2 / 8 / 6 0 3 3 1}$ placed in the lumbar region. This catheter can be 4F flush without any additional side holes. Catheters were sutured to the skin and covered by a Tegaderm transdermal patch (3M Health Care, St. Paul, Minn). The superior-most catheter was used as the infusion catheter; the second catheter at the level of the conus medullaris was the effusion catheter. The $4 \mathrm{~F}$ catheter was the pressure-monitoring catheter. The patient was transported to the operating room.

The aneurysm was resected through a standard thoracoabdominal incision. The chest was entered through the sixth intercostal space. The fifth rib was divided posteriorly. The diaphragm was completely divided, the left part of the colon, left kidney, tail of the pancreas, and spleen were mobilized, the left lung was deflated, and the entire thoracoabdominal aorta was exposed.

Shortly before the aorta was crossclamped, the cooling process began. The cooling system consisted of the following elements: (1) a Bentley BCR 3000/Baxter reservoir (Bentley Laboratories Division, Irvine, Calif.) containing normal saline solution; (2) a roller pump-Sarns model S10K2 blood pump (Sarns Inc., Ann Arbor, Mich); (3) a heat exchanger, which is a MYOtherm 41 Avecor cardioplegia delivery system (Avecor Cardiovascular Inc., Plymouth, Minn.). A CSZ Hemotherm cooler heater model No. 400M (Cincinnati Sub-Zero Products, Inc., Cincinnati, Ohio). The tubing for the drainage is a Medtronic cardioplegia delivery line (Medtronic, Inc., Minneapolis, Minn.) (Fig. 1).

The temperature of the coolant right before entering the subarachnoid space was $6^{\circ} \mathrm{C}$. The temperature of the draining solution was maintained at $20^{\circ}$ to $22^{\circ} \mathrm{C}$ with a delivery speed of 15 to $20 \mathrm{ml} / \mathrm{min}$, which corresponded to a spinal fluid pressure of no higher than $15 \mathrm{~cm} \mathrm{H}_{2} \mathrm{O}$.

The draining solution was discarded. The tubing sizes were $5 \mathrm{~F}$ inflow/outflow and $4 \mathrm{~F}$ pressure.

A 4000 unit dose of heparin was given intravenously. Once the temperature of the draining solution reached $20^{\circ} \mathrm{C}$, the aorta was crossclamped immediately distal to the takeoff of the left subclavian artery. A No. 20 Dacron graft was selected and an end-to-end anastomosis was constructed. The intercostal vessels above the T5 level were oversewn. The intercostal vessels below the T5 level, celiac access, superior mesenteric artery, and renal arteries were incorporated into the graft. A distal anastomosis was placed in the abdominal aorta above the bifurcation. After the flow through the graft was restored, the cooling was discontinued. The systemic temperature remained normothermic throughout the procedure. Catheters from the subarachnoid space were discontinued the following day. The patient recovered uneventfully, was extubated the following day without any neurologic complications, and was discharged 9 days later. 


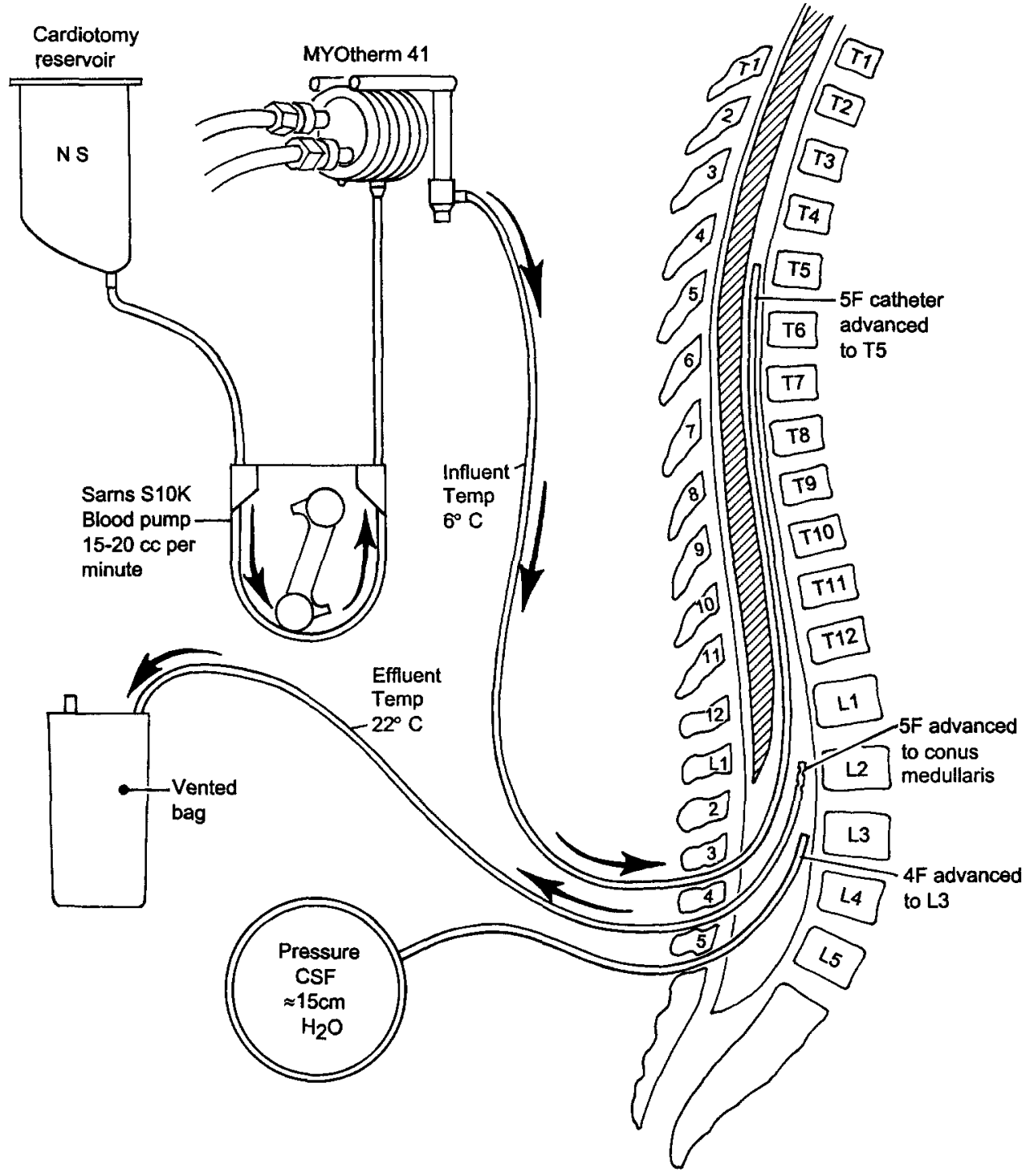

Fig. 1. Schematic view of the cooling circuit for the subarachnoid space. NS, Normal saline; CSF, cerebral spinal fluid.

Case 2. A 65-year-old woman also had a symptomatic thoracoabdominal aneurysm. The aneurysm was originating in the lower thoracic aorta. An angiogram showed that the origin of the aneurysm proximally was about 1 inch above the celiac access. It propagated throughout the abdominal aorta involving the entire abdominal segment. The proximal iliac arteries were ectatic but not truly aneurysmal. The protocol for spinal protection was exactly the same as in case 1 , although the operative procedure was obviously less extensive.

A thoracoabdominal incision was used through the eighth intercostal space. The aorta was crossclamped about 2 inches above the diaphragm. One pair of intercostals, celiac access, superior mesenteric artery, and renal arteries were incorporated as one island to the graft. The anastomosis distally was placed right at the bifurcation of the aorta. The patient recovered from the operation without any complications.

Conclusion. Selective cooling of the spinal cord with continuous lavage of cold normal saline solution demonstrates several important points:

1. Continuous lavage can be done in human beings. The low temperature of the coolant has not created a neurologic sequela per se. (Placement of the catheters to the subarachnoid space must be done by an experienced radiologist.)

2. Introduction of the cold normal saline solution to the subarachnoid space effectively and fairly rapidly reduces the temperature of the cerebrospinal fluid.

3. This method of spinal protection simplifies the operating procedure. (It eliminates the need for cumbersome bypasses.) 
4. Selective cooling of the spinal cord eliminates complications related to general hypothermia.

Although it is too soon to draw any conclusions regarding the effectiveness of this method of spinal protection, I believe that further clinical studies should be done. The results of the animal experiments have been encouraging., 2

Addendum. Since this manuscript was accepted for publication, this method of spinal protection has been used in two more patients requiring resection of arteriosclerotic thoracoabdominal aneurysm. The first patient was a 74-year-old man with a contained rupture of a thoracoabdominal aneurysm originating at the takeoff of the left subclavian artery down to the infrarenal aorta, where a graft had previously been placed for an abdominal aortic aneurysm. The crossclamp time was 46 minutes. The second patient was a 71-year-old man, also with a history of previously resected abdominal aortic aneurysm, who presented with a large asymptomatic thoracoabdominal aneurysm. The aneurysm involved the entire descending thoracic aorta and terminated right below the diaphragm. The aorta was crossclamped for 50 minutes. Both patients did well without any neurologic complications.

\section{REFERENCES}

1. Berguer R, Porto J, Fedoronko B, et al. Selective deep hypothermia of the spinal cord prevents paraplegia after aortic cross-clamping in the dog model. $\mathrm{J}$ Vasc Surg 1992;15:62-72.

2. Salzano R, Ellison L, Altonji $\mathbf{P}$, et al. Regional deep hypothermia of the spinal cord protects against ischemic injury during thoracic aortic cross-clamping. Ann Thorac Surg 1994;57:65-71.
3. Breckwoldt WL, Genco CM, Connolly RJ, et al. Spinal cord protection during aortic occlusion: efficacy of intrathecal tetracaine. Ann Thorac Surg 1991;51:95963.

4. Colon R, Frazier OH, Cooley DA, et al. Hypothermic regional perfusion for protection of the spinal cord during periods of ischemia. Ann Thorac Surg 1987;43: 639-43.

5. Crawford ES, Svensson LG, Hess KR, et al. A prospective randomized study of cerebrospinal fluid drainage to prevent paraplegia after high-risk surgery on the thoracoabdominal aorta. J Vasc Surg 1990;13:36-46.

6. Crawford ES, Mizrahi EM, Hess KR, et al. The impact of distal aortic perfusion and somatosensory evoked potential monitoring on prevention of paraplegia after aortic aneurysm operation. J THORAC CARDIOvasC SuRg 1988;95:357-67.

7. Crawford ES, Coselli JS, Safi HJ. Partial cardiopulmonary bypass, hypothermic circulatory arrest, and posterolateral exposure for thoracic aortic aneurysm operation. J Thorac CARdiovasc SuRg 1987;94:824-7.

8. Elmore JR, Gloviczki P, Harper CH Jr, et al. Spinal cord injury in experimental thoracic aortic occlusion: investigation of combined methods of protection. $\mathrm{J}$ Vasc Surg 1992;15:789-99.

9. Kouchoukos NT, Wareing TH, Izumoto $\mathrm{H}$, et al. Elective hypothermic cardiopulmonary bypass and circulatory arrest for spinal protection during operations on the thoracoabdominal aorta. J THORAC CARDIOvaSC SuRG 1990;99:659-64.

\title{
SUCCESSFUL OPERATION FOR SOLITARY FIBROUS TUMOR OF THE EPICARDIUM
}

\author{
Daisuke Segawa, MD, ${ }^{a}$ Hiroshi Yoshizu, MD, ${ }^{\mathrm{a}}$ Yoshiyuki Haga, MD, ${ }^{\mathrm{a}}$ Nobuo Hatori, MD, ${ }^{\text {a }}$ Susumu Tanaka, MD, \\ and Shinsuke Aida, MD, ${ }^{\mathrm{b}}$ Saitama, Japan
}

Solitary fibrous tumors, which mostly arise from the pleura, are not common, and lesions growing from the epicardium are extremely rare. We present a case of an epicardial solitary fibrous tumor that was found as an enlarged mediastinal shadow in a chest roentgenogram and was resected after $2 \frac{1 / 2}{2}$ years' observation.

From the Department of Surgery II $^{\mathrm{a}}$ and the Department of Laboratory Medicine, ${ }^{\mathrm{b}}$ The National Defense Medical College, Saitama, Japan.

J THORAC CARDIOVASC SuRg 1995;109:1246-8

Copyright (C) 1995 by Mosby-Year Book, Inc.

$0022-5223 / 95 \$ 3.00+0 \quad \mathbf{1 2 / 8 / 5 8 6 1 1}$
A 50-year-old woman was referred to the National Defense Medical College Hospital in November 1990 with a suspected ventricular aneurysm. Ultrasonic echocardiography revealed that it was a solid mass attached to the right ventricle with a stalk. Surgical resection was recommended to the patient, but she refused because of no subjective symptoms. She was thus followed up in the outpatient clinic.

About $2 \frac{1}{2}$ years later, the patient was admitted to our hospital because of dyspnea and palpitation in the right lateral decubitus position. A chest roentgenogram demonstrated enlargement of the mass after the $2 \frac{1}{2}$ years of follow-up (Fig. 1). The computed tomographic scan and the magnetic resonance image revealed that the lesion 